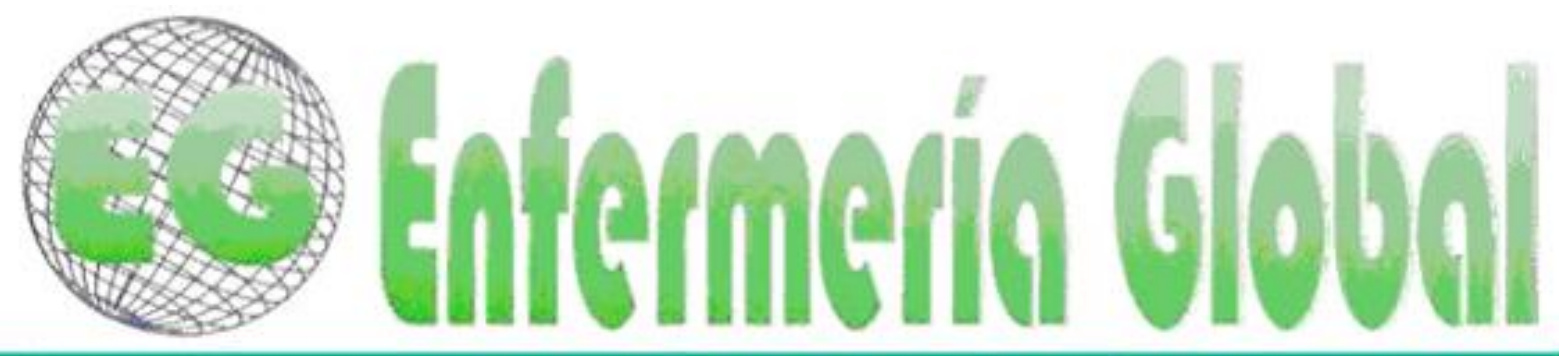

$N^{\circ} 35$

Revista electrónica trimestral de Enfermeria

Julio 2014

www.um.es/egloball

\title{
REVISIONES
}

\section{Acciones de enfermería para la prevención de la neumonía asociada a ventilación mecánica: revisión sistemática}

Ações de enfermagem para prevenção de pneumonia associada à ventilação mecânica: revisão sistemática

Nursing actions for the prevention of ventilator associated pneumonia: systematic review

\section{*Kluczynik Vieira, Caroline Evelin Nascimento **de Andrade, Paula Stefânia ${ }^{* * *}$ Enders, Bertha Cruz ${ }^{* * *}$ Coura, Alexsandro Silva ${ }^{* * * *}$ Dutra, Michelinne Oliveira Machado}

*Enfermera. Doctoranda del Programa de Postgrado en Enfermería de la Universidad Federal do Rio Grande do Norte (UFRN). E-mail: carolinekluczynik@gmail.com **Doctora en Enfermería. Docente del Programa de Postgrado en Enfermería de la Universidad Federal do Rio Grande do Norte. ${ }^{* * *}$ Doctor en Enfermería. Docente de la Universidad Estadual da Paraíba (UEPB). ${ }^{* * * *}$ Alumna de Máster del Programa de Postgrado en Salud Pública de la Universidad Estadual da Paraíba (UEPB). Brasil.

Palabras clave: Atención de enfermería; Neumonia asociada al ventilador; Prevención secundaria; Revisión

Palavras-chave: Cuidados de enfermagem; Pneumonia associada à ventilação mecânica; Prevenção secundária; Revisão

.Keywords: Nursing care; Pneumonia ventilator-associated; Secondary prevention; Review.

\section{RESUMEN}

Objetivo: Identificar acciones de enfermería implementadas en la prevención de la neumonía asociada a ventilación mecánica en los pacientes intubados en la Unidad de Cuidados Intensivos.

Método: Revisión sistemática en las bases: PubMed, BVS y Scielo utilizando los descriptores: "Atención de Enfermería" y "Neumonía Asociada al Ventilador". La muestra constaba de 13 artículos. La calidad interna se evaluó mediante la clasificación de Jadad.

Resultados: Los destaques fueron las acciones: elevación de decúbito de 30ㅜ; higiene oral; formación de las enfermeras; Protocolo Francés Destete de la Ventilación Mecánica dirigido a las enfermeras; proporción entre el número de enfermeras por turno, y evitar la reutilización de succión. 
Conclusión: Los cuidados de enfermería presentados deben ser utilizados en la prevención de la neumonía asociada a ventilación mecánica en los pacientes intubados en la Unidad de Cuidados Intensivos, ya que a través de los resultados de los artículos originales demostraron la eficacia de dichas acciones.

\section{RESUMO}

Objetivo: Identificar as ações de enfermagem implementadas na prevenção da pneumonia associada à ventilação mecânica nos pacientes intubados em Unidade de Terapia Intensiva.

Método: Revisão sistemática nas bases: PubMed, BVS e SciELO, através dos descritores: "Nursing Care" and "Pneumonia, Ventilator Associated". A amostra foi composta por 13 artigos. A qualidade interna foi avaliada pela classificação de Jadad.

Resultados: Destacaram-se as ações: elevação do decúbito superior a $30^{\circ}$; higienização oral; capacitação dos enfermeiros; Protocolo Francês de Desmame de Ventilação Mecânica dirigido às enfermeiras; dimensionamento de enfermeiros por plantão; e evitar a reutilização de equipamentos de aspiração.

Conclusão: Os cuidados de enfermagem apresentados devem ser empregados na prevenção da pneumonia associada à ventilação mecânica nos pacientes intubados em Unidade de Terapia Intensiva, uma vez que através dos artigos originais apresentados as referidas ações demonstraram eficácia.

\section{ABSTRACT}

Objective: To identify the nursing actions implemented in the prevention of ventilator-associated pneumonia in intubated and hospitalized patients in the Intensive Care Unit.

Method: systematic review of the databases: PubMed, VHL and Scielo using the keywords: "Nursing Care" and "Pneumonia, Ventilator-Associated". The sample consisted of 13 articles. The internal quality was assessed by Jadad classification.

Results: the highlights were the actions: elevation of decubitus than $30^{\circ}$; oral hygiene; training of nurses; French Protocol Weaning of Mechanical Ventilation directed to nurses; nurses proportion per shift; and avoiding the reuse of suctioning equipment.

Conclusion: nursing care provided must be used in the prevention of ventilator-associated pneumonia in patients intubated in the Intensive Care Unit, as shown through original articles that demonstrated the effectiveness of such actions.

\section{INTRODUCCIÓN}

La neumonía asociada a la ventilación mecánica (PAVM) es una infección nosocomial que ocurre en el parénquima pulmonar, se desarrolla 48 horas después del inicio de la ventilación mecânica (VM) y hasta 48 horas después de la extubación. Puede ser clasificada como precoz, hasta el cuarto día de intubación, o tardía, tras el quinto día ${ }^{(1)}$.

Se sabe que la PAVM está relacionada con el aumento de las tasas de morbimortalidad, días de ingreso y costos hospitalarios. La estimación de los costos imputables al paciente con PAVM en Europa es de aproximadamente $€ \$ 16.000$. E en los Estados Unidos el aumento se sitúa entre U\$10.000 a U\$40.000 por paciente ${ }^{(2)}$. 
Se hace hincapié en que la prevención a través de la higiene de las manos, el mantenimiento de decúbito elevado del paciente, la técnica adecuada de intubación y la aspiración traqueal entre otras precauciones, son fundamentales para reducir esta complicación infecciosa ${ }^{(3)}$.

Por lo tanto, las medidas de prevención son esenciales y deben aplicarse las estrategias de control para la normalización de la atención a los pacientes en riesgo. Se considera que la profilaxis de la NAVM es debida en gran parte a la enfermería, ya que en la unidad de estudio, el personal de enfermería tiene la mayoría de los procedimientos relacionados con la ventilación mecánica. Habiendo estudios pertinentes con el objetivo de evaluar la práctica de enfermería en la prevención de la enfermedad en pacientes con condiciones de salud graves, como intubados e ingresados en la Unidad de Cuidados Intensivos (UCI) ${ }^{(4)}$.

En este contexto, ¿cuáles son las medidas prevéntivas más eficaces que deben tomar los pacientes de enfermería en la UCl y sometidos a intubación? Con el fin de responder a esta pregunta, este estudio tuvo como objetivo identificar las acciones de enfermería realizadas en la prevención de la NAVM en pacientes hospitalizados en las UCl.

\section{MÉTODO}

Esta es una revisión sistemática realizada en enero de 2014. Los datos presentados provienen de los artículos publicados entre enero de 2001 y enero de 2014. La búsqueda de manuscritos se realizó a través de internet en PubMed, Biblioteca Virtual en Salud (BVS) y SciELO. Se utilizaron los Medical Subject Headings (MeSH): "Nursing care" y "Pneumonia, ventilator-associated", y sus términos correspondientes en los Descriptores en Ciencias de la Salud Headings (DeCS). Los criterios de inclusión fueron: ensayos clínicos en seres humanos, en lengua Inglesa, Española o Portuguesa, disponible en su totalidad, gratuitos, que abordasen acciones de enfermería para la prevención de la NAVM en pacientes hospitalizados en la UCI y sometidos a intubación.

Después de la búsqueda e identificación de los artículos se procedió a la lectura de los títulos y resúmenes, seleccionándose aquellos que atendieron los límites definidos. Al final de la selección, la muestra estuvo compuesta de trece artículos.

El proceso de recolecta y evaluación fue realizado por dos revisores independientes y ciegos. Para revisar los manuscritos se usó la escala de calidad de Jadad, que evalúa tres factores que influyen en la validez interna de un estudio: ¿El estudio se describió como aleatorio? ¿El estudio se describió como doble ciego? ¿Las pérdidas de seguimiento han sido descritas y tomadas de los pacientes del estudio? Cada ítem recibe un punto si la respuesta es "sí". Además, un punto puede añadirse o restarse, de acuerdo con la descripción adecuada del procedimiento de asignación o de enmascaramiento. Un estudio es considerado de mala calidad interna si recibe dos puntos o menos, siendo cinco la puntuación más alta. Esta clasificación analiza la sensibilidad de los elementos seleccionados, pero no se usa para excluir los estudios $^{(5)}$.

Para el análisis de datos, dos revisores leyeron de forma crítica los artículos seleccionados, extrayendo las unidades de interés para el estudio. Dichas unidades se estandarizaron y agruparon de acuerdo a la similitud de las ideas centrales que se 
presentan: características del artículo; ubicación durante el estudio; características de la población estudiada; diseño del estudio; objetivos; y resultados principales.

\section{RESULTADOS}

Después de la lectura de los títulos y los resúmenes se identificaron 63 artículos en las bases de datos de la BVS, Pubmed y SciELO. Fueron excluidos 48 estudios, de estos: 23 mostraron diferente enfoque del objetivo buscado (acciones de enfermería para la prevención de la NAVM en pacientes de UCI), 15 estaban dentro de la revisión de la literatura, y diez artículos no estaban disponibles, completos y on line. De los 15 artículos elegibles para el estudio, dos fueron excluidos debido a que estuvieron presentes en dos bases de datos (PubMed y BVS), siendo seleccionados al final 13 artículos para su análisis.

La Tabla 1 muestra la calidad interna de los estudios incluidos en esta revisión. Siguiendo esta clasificación ${ }^{(5)}$, los estudios con mayor calidad interna fueron: Munro et al. ${ }^{(2)}$, Prendergast et al. ${ }^{(6)}$ de Gan et al. ${ }^{(7)}$, una vez que han llegado a cuatro en la escala de calidad de Jadad. Seguido por Wei et al. ${ }^{(8)}$, Khalifehzadeh et al. ${ }^{(9)}$, Lyerla et al. ${ }^{(10)}$, Metheny et al. ${ }^{(11)}$ Tonnelier et al. ${ }^{(12)}$, que obtuvo puntuación de tres.

Tabla 1- Calidad interna de los estudios incluidos, evaluada por la escala de Jadad ${ }^{(5)}$. Natal/Río Grande do Norte, Brasil 2014.

\begin{tabular}{|c|c|c|c|c|}
\hline Estudios & $\begin{array}{l}\text { Selección } \\
\text { aleatoria * }\end{array}$ & $\underset{*}{\text { Doble ciego }}$ & 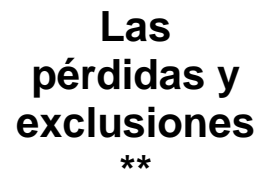 & $\begin{array}{c}\text { Escala de } \\
\text { calidad de } \\
\text { Jadad }\end{array}$ \\
\hline Weiet al. ${ }^{(8)}$ & SÍ/A & NO & 0 & 3 \\
\hline Khalifehzadeh et al. ${ }^{(9)}$ & Sí/A & NO & 0 & 3 \\
\hline Gonçalves et al. ${ }^{(13)}$ & Sí/B & NO & 0 & 2 \\
\hline Lyerla et al. ${ }^{(10)}$ & B & sí / A & 0 & 3 \\
\hline Munro et al. ${ }^{(2)}$ & sí / A & sí / A & $\begin{array}{c}53 \% \\
(288 / 537)\end{array}$ & 4 \\
\hline Prendergastet al. ${ }^{(6)}$ & Sí / A & Sí /A & $\begin{array}{c}34 \% \\
(26 / 76)\end{array}$ & 4 \\
\hline Bloos et al. ${ }^{(14)}$ & NO & $\mathrm{NO}$ & 0 & 1 \\
\hline Hugonnet et al. ${ }^{(15)}$ & $\mathrm{B}$ & sí / C & 0 & 1 \\
\hline Munro et al. ${ }^{(16)}$ & sí / A & $\mathrm{B}$ & $\begin{array}{c}44 \% \\
(29 / 66)\end{array}$ & 2 \\
\hline Metheny et al. ${ }^{(11)}$ & Sí / A & NO & 0 & 3 \\
\hline Grap et al. ${ }^{(7)}$ & sí / A & sí / A & $\begin{array}{c}76 \% \\
(210 / 276)\end{array}$ & 4 \\
\hline Tonnelier et al. ${ }^{(12)}$ & sí / A & $B$ & 0 & 3 \\
\hline Sole et al. ${ }^{(17)}$ & NO & NO & $\begin{array}{c}10 \% \\
(2-20)\end{array}$ & 1 \\
\hline
\end{tabular}

${ }^{*}$ Respuesta $\mathrm{SI}=1$ punto; Respuesta $\mathrm{NO}=0$ puntos. ${ }^{* *}$ Pérdidas $=$ cero vale 1 punto. $\mathrm{A}=$ Descripción adecuada (+1 punto); $\mathrm{B}=$ No descrita (0 puntos); $\mathrm{C}=$ Descripción inadecuada (-1 punto). 
La Tabla 2 presenta las principales características de las pruebas de las acciones de enfermería en la prevención de la NAVM en pacientes de UCI. De los 13 estudios seleccionados, siete se realizaron en los Estados Unidos ${ }^{(2,6-7,10-11,16-17)}$, tres en Europa ${ }^{(12-15)}$, uno en China ${ }^{(8)}$, uno en Brasil ${ }^{(13)}$ y uno en Irán ${ }^{(9)}$. Las publicaciones se concentraron en $s$ revistas de enfermería y cuidados intensivos. El diseño predominante fue el transversal ${ }^{(2,6,8-17)}$, aunque un estudio es longitudinal ${ }^{(7)}$. En cuanto a los criterios de inclusión, sólo un estudio seleccionó niños mayores de 10 años y adultos ${ }^{(14)}$, los demás sólo incluyeron a adultos y ancianos ${ }^{(2,6-13,15-16)}$.

Tabla 2-Características de los estudios de acciones de enfermería para la prevención de NAVM en pacientes intubados ingresados en UCI. Natal/RN, Brasil 2014.

\begin{tabular}{|c|c|c|c|c|c|c|}
\hline Referencias & Año & $\begin{array}{l}\text { Ubicació } \\
n\end{array}$ & Sujeto & $\begin{array}{l}\text { Eda } \\
d \\
e n \\
\text { año } \\
s\end{array}$ & Dibujo & Objetivos \\
\hline Weiet al. (૪) & 2013 & China & 615 & $\begin{array}{l}21- \\
92\end{array}$ & $\begin{array}{l}\text { Transvers } \\
\text { al }\end{array}$ & $\begin{array}{l}\text { Comparar los métodos } \\
\text { diferentes de enfermería en la } \\
\text { limpieza de la cavidad nasal de } \\
\text { pacientes en ventilación } \\
\text { mecánica. }\end{array}$ \\
\hline $\begin{array}{l}\text { Khalifehzadeh } \\
\left.\text { et al. }{ }^{9}\right)\end{array}$ & 2012 & Brasil & 35 & $\begin{array}{l}39- \\
42\end{array}$ & $\begin{array}{l}\text { Transvers } \\
\text { al }\end{array}$ & $\begin{array}{l}\text { Determinar la efectividad de la } \\
\text { estrategia educativa para } \\
\text { mejorar el rendimiento de } \\
\text { procedimientos preventivos de } \\
\text { neumonía asociada } \\
\text { ventilación mecánica. }\end{array}$ \\
\hline $\begin{array}{l}\text { Gonçalves } \\
\text { et al. }{ }^{(13)}\end{array}$ & 2012 & Irán & 54 & $\begin{array}{l}15- \\
65\end{array}$ & $\begin{array}{l}\text { Transvers } \\
\text { al }\end{array}$ & $\begin{array}{l}\text { Analizar los efectos de una } \\
\text { práctica de la higiene oral sobre } \\
\text { la incidencia de neumonía } \\
\text { asociada a ventilación mecánica } \\
\text { en pacientes en UCl. }\end{array}$ \\
\hline $\begin{array}{l}\text { Lyerla et } \\
\text { al. }{ }^{(10)}\end{array}$ & 2010 & $\begin{array}{l}\text { Estados } \\
\text { Unidos }\end{array}$ & 43 & $\begin{array}{l}39- \\
96\end{array}$ & $\begin{array}{c}\text { Transver } \\
\text { sal }\end{array}$ & $\begin{array}{l}\text { Observar si el cambio en el } \\
\text { ángulo de decúbito ayuda en la } \\
\text { prevención de NAVM. }\end{array}$ \\
\hline$\underset{(2)}{\text { Munro et al. }}$ & 2009 & $\begin{array}{l}\text { Estados } \\
\text { Unidos }\end{array}$ & 547 & $\geq 18$ & $\begin{array}{l}\text { Transver } \\
\text { sal }\end{array}$ & $\begin{array}{l}\text { Evaluar cuál es el mejor método } \\
\text { para la descontaminación en la } \\
\text { prevención oral de NAVM. }\end{array}$ \\
\hline $\begin{array}{l}\text { Prendergast } \\
\text { et al. }{ }^{6)}\end{array}$ & 2009 & $\begin{array}{l}\text { Estados } \\
\text { Unidos }\end{array}$ & 76 & $\geq 18$ & $\begin{array}{c}\text { Transver } \\
\text { sal }\end{array}$ & $\begin{array}{l}\text { Se refieren a la calidad de la } \\
\text { higiene bucal de los pacientes } \\
\text { con incidencia de NAVM. }\end{array}$ \\
\hline$\underset{(14)}{B l o o s}$ et al. & 2009 & Alemania & $\begin{array}{c}133 \\
\text { antes } \\
142 \\
\text { depois }\end{array}$ & $\geq 10$ & $\begin{array}{l}\text { Transver } \\
\text { sal }\end{array}$ & $\begin{array}{l}\text { Evaluar si la formación de } \\
\text { enfermeras UCl contribuye a la } \\
\text { reducción de las tasas de } \\
\text { NAVM. }\end{array}$ \\
\hline $\begin{array}{l}\text { Hugonnet et } \\
\text { al. }\end{array}$ & 2007 & Suiza & 2470 & $\begin{array}{l}48- \\
75\end{array}$ & $\begin{array}{l}\text { Transver } \\
\text { sal }\end{array}$ & $\begin{array}{l}\text { Determinar si la baja proporción } \\
\text { enfermera/paciente aumenta el } \\
\text { riesgo de NAVM. }\end{array}$ \\
\hline$\underset{(16)}{\operatorname{Munro}}$ et al. & 2006 & $\begin{array}{l}\text { Estados } \\
\text { Unidos }\end{array}$ & 66 & $\begin{array}{l}25- \\
93\end{array}$ & $\begin{array}{l}\text { Transver } \\
\text { sal }\end{array}$ & $\begin{array}{l}\text { Relacionar NAVM y la calidad de } \\
\text { la higiene oral. }\end{array}$ \\
\hline $\begin{array}{l}\text { Metheny et } \\
\text { al. (11) }\end{array}$ & 2006 & $\begin{array}{l}\text { Estados } \\
\text { Unidos }\end{array}$ & 360 & $\begin{array}{l}18- \\
95\end{array}$ & $\begin{array}{l}\text { Transver } \\
\text { sal }\end{array}$ & $\begin{array}{l}\text { Describir la frecuencia de } \\
\text { aspiración orotraqueal y NAVM, } \\
\text { vinculándolas con la posición de }\end{array}$ \\
\hline
\end{tabular}




\begin{tabular}{|c|c|c|c|c|c|c|}
\hline$\underset{(7)}{\text { Grap et al. }}$ & 2005 & $\begin{array}{l}\text { Estados } \\
\text { Unidos }\end{array}$ & 276 & $\begin{array}{l}25- \\
93\end{array}$ & Longitudinal & $\begin{array}{l}\text { Relacionar la elevación } \\
\text { decúbito e NAVM. }\end{array}$ \\
\hline $\begin{array}{l}\text { Tonnelier et } \\
\text { al. }{ }^{(12)}\end{array}$ & 2005 & Francia & 208 & $\begin{array}{l}18- \\
56\end{array}$ & $\begin{array}{c}\text { Transver } \\
\text { sal }\end{array}$ & $\begin{array}{l}\text { Determinar si el uso de de } \\
\text { protocolo francés de destete de } \\
\text { la ventilación mecánica, dirigid } \\
\text { a enfermeras, favorece } \\
\text { prevención de NAVM. }\end{array}$ \\
\hline Sole et al. & 2002 & $\begin{array}{l}\text { Estados } \\
\text { Unidos }\end{array}$ & 20 & $\geq 18$ & $\begin{array}{c}\text { Transver } \\
\text { sal }\end{array}$ & $\begin{array}{l}\text { Identificar si la reutilización de } \\
\text { circuito de aspiración y une } \\
\text { higiene oral están asociados cor } \\
\text { NAVM. }\end{array}$ \\
\hline
\end{tabular}

La metodología utilizada en los artículos y los principales resultados se presentan en la Tabla 3.El criterio más utilizado para el diagnóstico de NAVM fue la búsqueda de

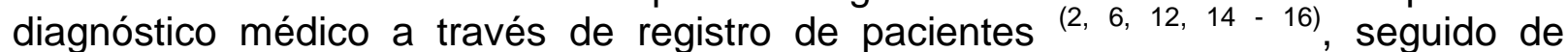
realización de cultivo microbiano ${ }^{(11,16,17)}$.

Tabla 3- Metodología y principales resultados de los estudios de enfermería en la prevención de NAVM en pacientes intubados. Natal/RN, Brasil 2014.

\section{Referencias}

Metodología

Resultados

Weiet al., 20123 grupos según el APACHE II. La diferencia fue (8) Aspiración de la cavidad bucal y estadísticamente significativa. En nasal. Aplicación de solución salina el grupo A, la incidencia de PAV nasal. Limpieza y aplicación de fue $36.76 \%$, grupo B fue de aerosol de solución salina. $30,24 \%$ y Grupo C fue $20,38 \%$.

Khalifehzadehe 2 grupos: enfermeras no aleatorias: La intervención fue eficaz para la t al., $2012^{(9)}$ intervención y comparación. limpieza de la lengua el $51 \%(p=$ Aplicación de la estrategia educativa 0,043 ), instalación del ventilador para la prevención de la PAV y $43 \%(p=0.048)$ y la secuencia posterior observación sistemática correcta de higiene bronquial (checklist). succión $13 \%(p=0.006)$.

Gonçalves al., $2012^{(13)}$

et 2 grupos: de intervención y control. La incidencia Prácticas de higiene bucal con asociada a la cepillado. Rutina de cuidado oral 2 mecánica, no difirió veces al día. La incidencia de PAV estadísticamente entre grupos de fue diagnosticada mediante intervención (37\%) y control puntuación de infección pulmonar $(48,1 \%), p=0.41$. clínica.

Lyerlaet al., Pacientes al azar según alternancia Cama con ángulción electrónica $2010^{(10)}$ en forma angular del decúbito. ayuda en las pautas en la Evaluación a través de la observación adhesión a directrices. Decúbito directa y entrevista.

en elevación promueve la prevención de las enfermedades respiratorias $(p=0.004)$.

Munroet al., $\quad 4$ grupos asignados al azar a la Clorhexidina sin cepillado $2009^{(2)} \quad$ higiene bucal: limpiar la clorhexidina disminuye los índices NAVM en $0.12 \%$, cepillado, cepillo + pacientes con neumonía en el clorhexidina y el grupo control. momento de la intubación $(\mathrm{p}=$ 0.006). 
Prendergastet al., $2009^{(6)}$

Blooset al. $2009^{(14)}$

A través de la guía de evaluación de $>$ Días intubación peor higiene la higiene Oral fue evaluada y bucal de los pacientes ( $\mathrm{p}=$ comparada con la incidencia de 0.004). Peor higiene oral se ha NAVM contenida en la tabla. asociado con tasas de NAVM.

Los registros médicos de pacientes $\mathrm{La}$ capacitación profesional recopilaron datos sobre: NAVM, días mejoró la adherencia a los de intubación y admisión, que se protocolos $(p=0,001)$, observada compararon antes y después de la a través de la disminución de formación de enfermeras.

intubación $(p=0,017)$ y hospitalización en unidad de cuidados intensivos $(p=0,04)$.

Hugonnetet al., Dimensión de enfermeros recogidos El aumento de enfermeras por $2007^{(15)}$ en nómina. El número de pacientes y paciente se asoció al centro el diagnóstico de NAVM a través de NAVM (cociente de riesgos = los registros. $0.42)$.

Munroet al., La higiene oral se evaluó mediante Higiene bucal deficiente se ha $2006^{(16)}$ observación directa. El diagnóstico asociado con la presencia de NAVM por registro. Colonización NAVM, debido al aumento de microbiana de la orofaringe y la colonización local por potenciales tráquea por cultivo después de 7 días patógenos $(p=0,01)$. de la intubación.

Methenyet al., La frecuencia de aspiración y NAVM La frecuencia de NAVM y $2006^{(11)}$ fueron determinados por el cultivo de aspiración fueron 31.3 y $88.9 \%$, la orofaringe y la tráquea. Y la respectivamente. El respaldo fue posición de decúbito por $30<$ factor de riesgo de observacióndirecta. aspiración $(\mathrm{p}=0.024)$ y neumonía $(0,018)$.

Grapetal., Puntuación clínica de la infección Elevação de decúbito inferior a $2005^{(7)}$ pulmonar fue utilizada para el $30^{\circ}$ estuvo relacionada con la diagnóstico de NAVM. La elevación tasa elevada de NAVM $(p=0,003)$. del decúbito fue verificada por sistema electrónico.

Tonnelieret al., PFDVM dirigido a enfermeras se La duración de la ventilación $2005^{(11)}$ comparó con el grupo control. mecánica $(p=0,02)$ y la duración Duración de la ventilación, duración de la estancia en la UCI $(p=0,02)$ de la estancia en la UCl y NAVM se fueron menores entre los obtuvieron del registro.

pacientes sometidos al protocolo.

Sole et al., Se recolectaron muestras del cultivo Después de 24 horas estaban $2002^{(17)}$ de la pared oral y equipos de succión todos los patógenos en la cavidad a las 24 y 48 horas después de la bucal y el $94 \%$ de equipos de intubación.

succión contiene los mismos agentes patógenos.

PFDVM = protocolo francés del destete de la ventilación mecánica.

Con respecto a las acciones de enfermería tomadas en la prevención de la NAVM, se observa que: tres estudios informaron de elevación de decúbito y de acuerdo con los resultados obtenidos, la elevación de la cama superior a $30^{\circ}$ se debe utilizar $(7,10-11)$; cuatro estudios evaluaron la higiene oral, concluyeron que el protocolo más eficaz para prevenir la aparición de infecciones sistémicas, siendo la clorhexidina farmacológica la más eficaz comparada con solución salina ${ }^{(2,15,16,9)}$; un estudio analizó la capacitación de los enfermeros, sus resultados indicaron que esa estrategia tuvo efecto positivo en el pronóstico del paciente ${ }^{(14)}$, un estudio comparó diferentes métodos de higienización utilizados por la enfermería en la cavidad nasal de pacientes bajo ventilación mecánica mostrando que la aplicación de solución salina 
después de la limpieza de la cavidad nasal es más eficaz en la prevención de la NAVM ${ }^{(8)}$.

Otro estudio comparó la efectividad de la estrategia educativa para mejorar el rendimiento de los procedimientos de prevención de la PAV y encontró que la mayoría de las medidas recomendadas para reducir PAV relacionadas con la posición de la cabecera de la cama, la higiene bronquial y oral, la administración de la dieta y las medidas de gestión de circuitos del ventilador no se siguieron ${ }^{(13)}$.

Otro estudio examinó la aplicabilidad del Protocolo Francés de Destete de la Ventilación Mecánica dirigido a enfermeras y concluyó que su uso reduce la carga financiera y la asistencia en el pronóstico del paciente, ya que los participantes tuvieron menos días de hospitalización y uso de ventilación mecánica ${ }^{(12)}$; otro estudio examinó el número de enfermeros concluyendo que cuanto menor es la cantidad de enfermeros en la atención, mayor es la incidencia de la NAVM ${ }^{(15)}$; Finalmente, un artículo mostró que la reutilización del equipo de succión debe ser evitado, excepto después de la esterilización ${ }^{(17)}$.

\section{DISCUSIÒN}

El riesgo de neumonía es 10 a 20 veces mayor en la UCI. Este entorno es un epicentro de resistencia bacteriana y riesgo de infección, debido a la condición clínica de los internos y la variedad de procedimientos invasivos. Además, cuando se somete a la ventilación mecánicael riesgo a desarrollar NAVM es 7\% a 40\%, ya que el tubo endotraqueal proporciona una superficie inferior de las bacterias, que se adhieren y crecen para formar biopelículas que pueden ser absorbidas por el tracto respiratorio inferior ${ }^{(18)}$.

Para la prevención de la NAVM, se han desarrollado varios estudios para identificar las mejores técnicas que se utilizarán, sobre todo cuidando acciones porque las enfermeras son responsables de las necesidades continuas, directas y básicas de los pacientes con cuidado ${ }^{(15)}$. Aunque parte de las acciones empleadas en la prevención de la NAVM son comunes a todos los pacientes, graves o no, acaban en negligencias por ej. De la higiene oral.

Estudios mostraron que los enfermeros y técnicos de enfermería informaron de que la higiene oral es difícil, desagradable, con la posibilidad de mover el tubo endotraqueal y que no es una acción de enfermería tan importante ${ }^{(19)}$. Este hallazgo es preocupante, ya que la falta de higiene oral se asocia directamente con NAVM debido al aumento de la colonización local de patógenos potenciales ${ }^{(6,13,16)}$.

Un estudio en los Estados Unidos examinó los efectos de cepillado con hisopo y asociaciones de clorhexidina, en el desarrollo de NAVM. La muestra estuvo constituida por 547 pacientes sin neumonía en el momento de la intubación, fueron asignados al azar en cuatro grupos: hisopo bucal con clorhexidina 2 veces al día; cepillado 3 veces al día; clorhexidina y cepillado de los dientes; y el grupo de control. Los resultados mostraron que la clorhexidina redujo la incidencia de la VAP, el cepillado no tuvo ningún efecto significativo ni aumentó el efecto de la clorhexidina. Por lo tanto, los autores concluyeron que el uso tópico de clorhexidina y el cepillado de dientes no reducenNAVM. ${ }^{(2)}$. 
Otra atención de enfermería que merece mención es el mantenimiento de la cabeza elevada del paciente. Los resultados de los estudios analizados convergen en la siguiente constatación: el ángulo de más de $30^{\circ}$ promueve el intercambio de gases desde el paciente y evita $\mathrm{NAVM}^{(7,10-11)}$, ya que la elevación del respaldo disminuye la posibilidad de aspiración en $88,9 \%{ }^{(11) .}$.'

Los Centers for Disease Control and Prevention (CDC) recomiendan el decúbito de pacientes que utilizan VM entre $30^{\circ}$ y $45^{\circ}$. Recomendación avalada por el Institute for Healthcare Improvemente y la American Association of Critical-Care Nurse. La recomendación del Canadian Care Trials Group es similar: los pacientes con ventilación mecánica no pueden permanecer por debajo de los $30^{\circ}$ de posición ${ }^{(10)}$. Estudio conducido con 276 pacientes adultos intubados hospitalizados en la UCl, reveló que las posiciones de décubito inferior $30^{\circ}$ se asocia directamente con NAVM( $p$ $=0,003)^{(7)}$.

Otra estrategia empleada es el uso de protocolos para el destete de VM. Un estudio francés encontró que su uso ha ayudado a reducir la duración de la VM y la duración de la estancia en la UCl, en comparación con los sujetos control ${ }^{(12)}$. Al analizar los estudios internacionales se percibe que la enfermería se ha preocupado por mejorar su práctica. Sin embargo, la laguna existente en los ensayos clínicos llevados a cabo por las enfermeras brasileñas todavía refuerzan el distanciamiento con el manejo de respiradores, ventilación, teniendo publicados en su mayoría estudios teóricos ${ }^{(20)}$. En esta revisión sistemática un ensayo clínico brasileña relacionado con la temática abordada fue encontrado en las bases de datos utilizadas.

Un estudio en China con 615 sujetos de entre 21 y 92 años ingresados en la UCI y en ventilación mecánica comparó los diferentes métodos de higiene de la enfermería en la cavidad nasal de los pacientes con ventilación mecánica y encontró que la limpieza con solución salina después de la limpieza de la cavidad nasal es más eficaz en la prevención de la $\mathrm{PAV}^{(8)}$.

EI CDC los clasifica en categorías de factores de riesgo para la neumonía nosocomial: factores del huésped, cirugía, medicamentos, dispositivos invasivos y equipo respiratorio. Estudio en EE.UU. examina la reutilización de equipos para la aspiración y llegó a la conclusión de que deben ser esterilizados después de cada uso. Después de 24 horas de intubación, todos los participantes tenían sus patógenos que colonizan la cavidad oral y $94 \%$ de los sistemas de aspiración traqueal fueron colonizados con los mismos microorganismos ${ }^{(17)}$.

Estudio de cohorte realizado en Suiza trató de determinar la influencia del número de enfermeros en la tasa de NAVM y llegó a la conclusión de que el aumento de los enfermeros se asoció con una disminución de la NAVM ${ }^{(15)}$. Sin embargo, otros autores han sugerido que la formación de los enfermeros está afectando a la calidad de la atención. Estudio alemán evaluó las tasas de NAVM, la duración de la hospitalización y VM antes y después de la capacitación profesional de las enfermeras de la UCI. Sus resultados mostraron que la formación mejoró la adherencia a cuidar protocolos, probadas por la disminución de los días en la UCI y VM, sin embargo no hubo diferencias significativas en la frecuencia de NAVM ${ }^{(14)}$.

Otro estudio realizado en Brasil analizó la efectividad de la estrategia educativa para mejorar el desempeño del personal de enfermería en la realización de procedimientos de prevención de la NAVM y concluyó que la intervención fue efectiva para la limpieza 
de la lengua, del conjunto del ventilador y el orden correcto de tubo - nariz - boca en la higiene bronquial ${ }^{(13)}$.

En esta revisión, los estudios con mejor evaluación de la escala de Jadad ${ }^{(5)}$, son los ensayos clínicos aleatorizados, controlados, doble ciego ${ }^{(2,6,7)}$. Pero hay que subrayar que las evidencias no sólo dependen del tipo de diseño de investigación. Por otra parte, la búsqueda de evidenciaa sobre las prácticas asistenciales específicas, como requiere el área de control de la infección, exigen la necesidad de cumplir los criterios de elaboración de los ensayos clínicos, el control de varios factores de riesgo para la infección, lo que puede afectar a los resultados además de aquel bajo intervención ${ }^{(21)}$.

La demostración de que las acciones de enfermería pueden ayudar o dificultar la recuperación de los pacientes críticos, conduce a la necesidad de desarrollo de los ensayos clínicos, con el objetivo de mejorar las técnicas y desarrollar nuevas tecnologías para prestar una asistencia más eficaz de la enfermería proporcionando un mejor pronóstico y comodidad a los pacientes críticamente enfermos ${ }^{(22)}$.

La falta de homogeneidad entre las acciones de enfermería, hace que no sea posible llevar a cabo un meta-análisis. Por otra parte, una limitación de este estudio es la inclusión de sólo los artículos de libre acceso a través de Internet, teniendo en cuenta que algún trabajo importante para la temática puede no haber sido considerado.

\section{CONCLUSIÒN}

La mayoría de los estudios sugiere que la elevación del decúbito más de $30^{\circ}$ y la higiene bucal son los cuidados de enfermería que se deben emplear en la prevención de la NAVM en pacientes de UCI intubados. Es de destacar que los estudios analizados indicaron que se necesitan más investigaciones para identificar el mejor ángulo de la cama y uso de camas de elevación electrónica para incentivar a las enfermeras a unirse a este tipo de protocolos.

En la higiene oral, se observó que el uso tópico de clorhexidina redujo la colonización de la cavidad oral y redujo la incidencia de la NAVM. Se entiende, además, que este procedimiento es seguro y bien tolerado según los estudios analizados. Al comparar el costo de su uso con el aumento de la carga financiera de un NAVM, la aplicabilidad de la clorhexidina es una medida de bajo costo. Sin embargo, se necesitan más investigaciones para determinar la concentración óptima, forma de presentación, frecuencia y técnica de aplicación.

Por último, también se indicaron medidas eficaces: la formación de las enfermeras; el uso del Protocolo Francés de Destete de la Ventilación Mecánica dirigido a las enfermeras; dimensionamiento de las enfermeras; y evitar la reutilización del equipo de succión.

\section{REFERENCIAS}

1. Tantipong $\mathrm{H}$, Morkchareonpong $\mathrm{C}$, Jaiyindee $\mathrm{S}$, Thamlikitkul V. Randomized controlled trial and meta-analysis of oral decontamination with $2 \%$ chlorhexidine solution for the prevention of ventilator-associates pneumonia. Infect Control Hosp Epidemiol. 2008; 29(2):131-6. 
2. Munro CL, Grap MJ, Jones DJ, McClish DK, Sessler CN. Chlorhexidine, toothbrushing, and preventing ventilator-associated pneumonia in critically ill adults. Am J Crit Care. 2009; 18(5):427-38.

3. Agência Nacional de Vigilância Sanitária-ANVISA. Unidade de Investigação e Prevenção das Infecções e dos Eventos Adversos. Gerência Geral de Tecnologia em Serviços de Saúde- GGTES. Infecções do trato respiratório: orientações para prevenção de infecções relacionadas à assistência à saúde. 2011.

4. Gonçalves FAF, Brasil VV, Ribeiro LCM, Tipple AFV. Ações de enfermagem na profilaxia da pneumonia associada à ventilação mecânica. Rev. Acta paul. enferm. 2012. 25 (1). 101-7.

5. Jadad AR, Moore RA, Carroll D, Jenkinson C, Reynolds DJ, Gavaghan DJ. Assessing the quality of reports of randomized clinical trials: is blinding necessary? Control Clin Trials. 1996; 17(1):1-12.

6. Prendergast V, Hallberg IR, Jahnke H, Kleiman C, Hagell P. Oral health, ventilatorassociated pneumonia, and intracranial pressure in intubated patients in a neuroscience intensive care unit. Am J Crit Care. 2009; 18(4):367-76.

7. Grap MJ, Munro CL, Hummel RS, Elswick Jr, McKinney JL, Sessler CN. Effect of backrest elevation on the development of ventilator-associated pneumonia. Am J Crit Care. 2005; 14(4):324-33.

8. Wei L, Quin G, Yang X, Hu M, Jiang F, Lai T. A new nasal cavity nursing methods application in patients with mechanical ventilation. Pakistan Journal of Medical Sciences. 2013; 29 (4); 977-981. Khalifehzadeh A, Parizade A, Hosseini A, Yousefi $\mathrm{H}$. The effects of an oral care practice on incidence of pneumonia among ventilator patients in ICUs of selected hospitals in Isfahan, 2010. Iranian Journal of Nursing and Midwifery Research. 2012; 17 (3); 216-9.

9. Lyerla F, LeRouge C, Cooke DA, Turpin D, Wilson L. A nursing clinical decision support system and pontential predictors of head-of-bed position for patients receiving mechanical ventilation. Am J Crit Care. 2010; 19(1):38-47.

10. Metheny NA, Clouse RE, Chang Y, Stewart BJ, Oliver DA, Kollef MH. Tracheobronchial aspiration of gastric contents in critically ill tube-fed patients: frequency, outcomes, and risk factors. Crit Care Med. 2006; 34(4):1007-15.

11. Tonnelier JM, Prat G, Gal GL, Gut-Gobert C, Renault A, Boles JM, et al. Impact of a nurses' protocol-directed weaning procedure on outcomes in patients undergoing mechanical ventilation for longer than 48 hours: a prospective cohort study with a matched historical control group. Crit Care. 2005; 9(2):R83-9.

12. Gonçalves FAF, Brasil VV, Minamisava R, Caixeta CR, Oliveira LMAC, Cordeiro JABL. Eficácia de estratégias educativas para ações preventivas da pneumonia associada à ventilação mecânica. Rev. Esc. Anna Nerry. 2012.16 (4) 802 808.

13. Bloos F, Müller S, Harz A, Gugel M, Geil D, Egerland K, et al. Effects of staff training on the care mechanically ventilated patients: a prospective cohort study. $\mathrm{Br} \mathrm{J}$ Anaesth. 2009; 103(2):232-7.

14. Hugonnet S, Uçkay I, Pittet D. Staffing level: a determinant of late-onset ventilator-associated pneumonia. Crit Care. 2007; 11(4):1-7.

15. Munro CL, Grap MJ, Elswick Jr, McKinney J, Sessler CN, Hummel RS. Oral health status and development of ventilator-associated pneumonia: a descriptive study. Am J Crit Care. 2006; 15(3):452-60.

16. Sole ML, Poalillo FE, Byers JF, Ludy JE. Bacterial growth in secretions and on suctioning equipment of orally intubated patients: a pilot study. Am J Crit Care. 2002; 11(2):140-9. 
17. Oliveira LCBS, Carneiro PPM, Fischer RG, Tinoco EMB. A presença de patógenos respiratórios no biofilme bucal de pacientes com pneumonia nosocomial. Rev Bras Terap Intens. 2007; 19(4):428-33.

18. Westphal MRA. Avaliação do conhecimento e atitudes dos profissionais das Unidades de Terapia Intensiva sobre a relação entre higiene bucal, doença periodontal e pneumonia [dissertação]. São Paulo(SP): Centro de Pesquisas Odontológicas, Faculdade de Odontologia de São Leolpoldo Mandic; 2008.

19. Marinho BVS, Araújo ACS. O Uso dos enxaguatórios bucais sobre a gengivite e o biofilme dental. Intern J Dentistry. 2007; 6(4):124-31.

20. Nepomuceno RM, Silva LD. Pesquisa bibliográfica dos sistemas de vigilância em ventilação mecânica: o estado da arte na enfermagem. Rev Eletr Enf. 2007; 9(1):191-9.

21. Ramritu P, Halton K, Cook D, Whitby M, Graves N. Catheter-related bloodstream infections in intensive care units: a systematic review with meta-analysis.

J Adv Nurs. 2008; 62(1):3-21. 